\title{
Neue Konzepte der Pathologie im 17. Jahrhundert
}

\author{
Von Nikolaus Mani
}

Die spektakulären anatomischen und physiologischen Entdeckungen im 17. Jahrhundert (Blutkreislauf, Lymphgefäßsystem, Drüsenstruktur usw.) haben die Auffassungen und Kenntnisse über den Bau und die Verrichtung des Organismus grundlegend geprägt ${ }^{1}$. Daneben ist aber auch die Pathologie von der anatomischen und physiologischen Methodik und Denkweise erfaßt worden. Diese neuen Konzepte der Pathologie sollen an einigen Beispielen gezeigt werden.

William Harvey erläuterte in seinem Buch De motu cordis die Pathogenese des Fieberanfalls beim Dreitagefieber. Im Initialstadium geschieht folgendes. Das Fiebergift erreicht auf der Kreislaufbahn Herz und Lungen und verharrt dort eine Zeit lang. Dabei wird das Lebenszentrum beschwert, das Blut wird zur Lunge hin gestoßen, durchfließt aber dieselbe nicht, wie Harvey bei der pathologischen Sektion beobachtet haben will. Die Patienten sind von Atemnot bedrängt, sie keuchen, verharren unbeweglich, der Puls ist klein, schnell und unregelmäßig ${ }^{2}$. Im zweiten Stadium, dem eigentlichen Paroxysmus, steigert sich die Lebenswärme zur Fieberhitze, die träge eingedickte Blutmasse verflüssigt sich und wird dann durch die eröffneten arteriellen Blutwege kräftig in den ganzen Körper getrieben. Dabei wird das Krankheitsgift von der Heilkraft der Natur überwunden und ausgestoßen ${ }^{3}$.

Hier interpretiert Harvey den Paroxysmus des Tertianfiebers als Folge einer Kreislaufstörung.

Die Kenntnis des Blutkreislaufs, so führt Harvey aus, erhellt auch den Mechanismus der Verteilung ansteckender Stoffe im Organismus. Das Schlangengift etwa, das Luesvirus, die contagiöse Materie der Tollwut dringen manchmal ohne äußere Verletzung nach bloß oberflächlichem Kontakt in die Venenmünder ein und gelangen mit dem venösen Blut zum Herzen und von dort in den ganzen Körper ${ }^{4}$. Auch äußerlich verabreichte Arzneimittel werden durch die Venenöffnungen aufgesaugt und durch den zirkulierenden Blutstrom in den Körper verteilt. Auf die Haut geriebene spanische Fliege treibt den Harnfluß an, Knoblauch der auf die Fußsohlen gelegt wird, fördert die Expektoration ${ }^{5}$.

Jan de Wale, der hervorragende experimentelle Erforscher der Blutzirkulation und der lymphatischen Resorption wies nachdrücklich auf die 
Klärung physiopathologischer Mechanismen aufgrund der Zirkulationslehre hin. Nach einer straffen Ligatur des Armes erfolgt die Atrophie des Gliedes durch mangelnden arteriellen Zufluß und nicht, wie man bis dahin vermutet hatte, durch die Behinderung des zur Peripherie strömenden venösen Nährblutes ${ }^{6}$. Bei Krampfadern des Ober- und Unterschenkels sowie bei Hämorrhoiden sackt das Blut aus den großen oberen Venenästen nach unten ab. De Wale führte aus ${ }^{7}$ : «Aber es handelt sich um eine unnatürliche Bewegung des Blutes, weil die erschlafften Venen das Blut nicht aufwärts führen, sondern stauen, und so die Flüssigkeit durch ihre Eigenschwere sich der naturgemäßen Aufwärtsbewegung widersetzt und absinkt. Sie sammelt sich dann in den unteren Venen an und bewirkt deren Überdehnung und schließlich Krampfadern, zumal fortwährend neues Blut aus den Arterien hinzutritt.»

Die Entdeckung des Ductus Pancraeticus und die neue Deutung des Pancreasorganes als exkretorische Drüse (glandula conglomerata) verlieh der Pathologie des Organes ein neues Relief ${ }^{8}$. Reiner De Graaf ${ }^{9}$ teilte die Krankheiten des Pancreas aufgrund eigener und fremder Beobachtungen in zwei Hauptgruppen ein: Erkrankungen der Drüsensubstanz und pathologische Veränderungen des Bauchspeichels (morbi quibus pancreatis substantia infestatur ... vitia primaria quae in eius succum incidere possunt ${ }^{10}$. Die Erkrankungen des Parenchyms sind vielfältig und umfassen Schwellungen, Verhärtungen, Abszesse, Steinbildungen, karzinomatöse Wucherungen mit beträchtlichen Ulzerationen und invasiver Zerstörung der umgebenden Organe $^{11}$. Die pathologischen Veränderungen des Pancreassaftes sind quantitativer und qualitativ-chemischer Art ${ }^{12}$.

Das Pankreas kann zu viel oder zu wenig Bauchspeichel sezernieren, wobei die Beschaffenheit des Blutes, aus dem der succus pancreaticus abgesondert wird, eine ausschlaggebende Rolle spielt. Ein Übermaß an sauren Speisen fördert die Sekretion des Bauchspeichels ${ }^{13}$. Wenn das Blut viel zähflüssige Bestandteile enthält, können die Bauchspeichelgänge verstopft und der Sekretabfluß behindert werden ${ }^{14}$. Der gewöhnlich leicht sauer reagierende Bauchspeichel kann von seiner physiologischen Beschaffenheit abweichen, wobei er zu stark angesäuert wird oder auch zu wenig saure Bestandteile enthält ${ }^{15}$.

Vor der Entdeckung des Bauchspeichelganges durch Johann Georg Wirsung im Jahre 1642 hatte man das Pankreas als ein schützendes und stützendes Kissen für die Blutgefäße der oberen Bauchgegend betrachtet. An pathologischen Prozessen des Pankreas wurden Schwellungen, Abszesse, 
Verhärtungen, krebsige Wucherungen beschrieben. Als in den fünfziger und sechziger Jahren des 17. Jahrhunderts das Pankreas den exokrinen Drüsen zugeordnet wurde, änderte sich die Lehre der pankreatischen Erkrankungen schlagartig. Pankreaspathologie wurde zur Pathologie einer Drüse mit äußerer Sekretion. Man unterschied nun morphologische Veränderungen der Drüsensubstanz, Obstruktionen der Ausführungsgänge und chemische Alterationen des Bauchspeichels.

Wepfers Studie über die Apoplexie verkörpert in exemplarischer Weise die neue auf der Kreislauflehre und der pathologischen Anatomie basierende Krankheitslehre ${ }^{16}$. Bisher hatte man die Verstopfung der Hirnhöhlen als Ursache des Schlagflusses betrachtet. Diese alten liebgewordenen Vorstellungen, so stellte Wepfer fest, müssen gänzlich aufgegeben werden ${ }^{17}$. Der Schlagfluß ist keine ventrikuläre Obstruktion, er entsteht vielmehr aus einer cerebralen Zirkulationsstörung. Das Gehirn ist eines der am kräftigsten durchbluteten Organe ${ }^{18}$. Auch für das Gehirn gilt die Kreislauflehre. Durch mächtige Arterien, die sich in haardünne Gefäße aufteilen, strömt das Blut in die cerebrale Substanz und fließt über die venösen Sinus und die Halsvenen ins Herz zurück. Im Mark des Gehirns werden aus dem arteriellen Blute die Spiritus animales abgesondert; sie strömen in die Nerven ein und vermitteln Bewegung und Empfindung. Wepfer stellte fest: «Eine Apoplexie entsteht vor allem entweder in Folge einer unterbrochenen Zufuhr arteriellen Blutes zum Gehirn oder durch den behinderten Abfluß des spiritus animalis aus Groß- und Kleinhirn in die Nerven und das Rückenmark, oder auch beim Zusammentreffen beider Ursachen.» ${ }^{19}$ Die Unterbrechung der Blutzufuhr geschieht z. B. durch die Ruptur von Gehirnarterien ${ }^{20}$. Dabei tritt eine Hirnblutung auf, die Produktion der Spiritus animales hört auf und die Hirnsubstanz wird zusätzlich komprimiert ${ }^{21}$. Die Verstopfung der Karotis mit schleimig-fibrösen Blutgerinnseln führt ebenfalls zu cerebraler Ischämie ${ }^{22}$ und schließlich kann eine Obstruktion der Hirnarteriolen mit viskösem Blut zum Schlagfluß führen ${ }^{23}$. Je nach dem mehr oder weniger Arteriolen verstopft sind und entsprechend dem Ausmaß der Obstruktion sind die neuropsychischen Funktionen mit wechselnder Intensität betroffen, wobei die klinische Skala von Sprechstörungen über eine Hemiplegie bis zur totalen Apoplexie reicht ${ }^{24}$.

Wepfers Studie über die Apoplexie zeigt klar die neuen methodischen Elemente und Konezpte der naturwissenschaftlich orientierten Pathologie des 17. Jahrhunderts. Sie basiert auf anatomischen Studien der cerebralen Gefäße, auf den Befunden pathologischer Sektionen bei apoplektischem 
Insult und auf physiopathologischen Konzepten im Rahmen der Kreislauflehre. Alle diese Beobachtungen, Kenntnisse und theoretischen Überlegungen werden mit den klinischen Erscheinungen korreliert und zu einem völlig neuen Bild der Pathogenese des Schlagflusses verschmolzen. Wepfers Arbeit bildet den Übergang von der klassischen Humoral- zur modernen Kreislaufpathologie. Apoplexie entsteht nicht aus einer Verstopfung der Hirnventrikel mit viskösen Säften, sie ist vielmehr Folge einer zerebralen Ischämie.

Die pathologische Anatomie, meist Anatomia practica genannt, die sich als morphologische Disziplin auf konkrete und der unmittelbaren Beobachtung zugängliche Daten stützte, bildete den Kern der neuen Krankheitslehre. William Harvey wies darauf hin, daß die Eröffnung der Leichen nach tödlichen Leiden erst eine wissenschaftliche Pathologie (pathologia philosophica) begründe ${ }^{25}$. Die pathologischen Veränderungen, insbesondere bei chronischen Krankheiten seien manchmal monströser Natur. Harvey führte aus: Die Sektion einer Leiche, die von einem Patienten stamme, der an Auszehrung, an einem chronischen Leiden oder an Vergiftung zugrunde gegangen sei, bringe der Heilkunde mehr Nutzen als die Anatomie von zehn gehängten Delinquenten ${ }^{26}$.

Eine kurze präzise Fassung der neuen Pathologie gelang Johann Conrad Peyer in seinem Jugendwerk «Methode der Klinisch-anatomischen Krankengeschichten» (Methodus historiarum anatomico-medicarum, Paris 1678). Peyers Programm enthielt folgende Punkte ${ }^{27}$ :

1. Genaue klinische Schilderung unter Einbezug der gesamten Lebensgeschichte in gesunden und kranken Tagen.

2. Exakte pathologische Sektion durch einen Arzt, der die normale Anatomie vollständig beherrscht.

3. Deutung des pathogenetischen Prozesses aufgrund der klinischen und pathologisch-anatomischen Befunde und im Lichte der neuen anatomophysiologischen Erkenntnisse des 17. Jahrhunderts. So finde man den Sitz und die Ursachen (sedes et causae) der Krankheiten ${ }^{28}$.

Als illustrierendes Beispiel seiner Methode beschreibt Peyer einen Fall von Aszites, der aus der Verwachsung von Herz und Herzbeutel entstanden war (De ascite vitio cordis genito) ${ }^{29}$.

Bei einem 16 jährigen Jüngling, der seit jeher an Atemnot gelitten hatte, nahmen die Atembeschwerden im letzten Lebensjahre bedrohlich zu. Der Unterleib, die Hoden, Oberund Unterschenkel schwollen an, bei Fingerdruck blieb eine Delle zurück. Der Puls war schwach, klein und geschwind. Die Schwellungen wurden immer größer, das Körperge- 
wicht nahm zu, und die Atmung wurde unter lästigem Husten sehr beschwerlich. Der junge Patient starb eines qualvollen Todes. Bei der Sektion strömte nach Eröffnung der Bauchdecken Flüssigkeit wie aus einem Quell hervor; die Baucheingeweide waren nicht verändert. Der Sitz der Krankheit lag im Brustkorb. Lungen und Herzbeutel waren verklebt und auch das Herz war mit dem Perikard verwachsen. Das Herz hatte sich vergeblich aus seinen Fesseln zu befreien versucht und war in diesem aussichtslosen Bemühen ermattet. Das Blut stockte, Gerinnsel bildeten sich in der Herzhöhle, und die stagnierende Blutmasse führte zu Aszites.

In dieser Beschreibung Peyers finden wir charakteristische Elemente der neuen Pathologie. Er verbindet die klinische Beobachtung mit dem autoptischen Befund und deutet die Krankheit als Zirkulationsstörung. Die Herzschwäche entsteht aus der Behinderung der Herztätigkeit durch die Verwachsung des Herzens mit dem Perikard und mit den Lungen; die erlahmende Herzkontraktion führt zu Blutstauung und zu Aszites.

Die theoretische Begründung der neuen Pathologie lieferten in kraftvollen Zügen und voll optimistischer Grundstimmung Niels Stensen ${ }^{30}$ und Marcello Malpighi ${ }^{31}$. Stensen führte aus: Die Lehre des gesunden und kranken Körpers muß von Grund auf neu erarbeitet werden. Der Körper ist, wie Decartes es wollte, eine Maschine. Krankheit bedeutet Störung der Körpermaschine. Therapie besteht in der Reparatur der aus den Fugen geratenen Maschine. Der Arzt kann aber erst reparieren, wenn er den Bau dieser nach physikalischen Gesetzen funktionierenden Maschine kennt ${ }^{32}$. Deshalb lautet der Forschungsauftrag: Zerlegung des organischen Uhrwerks in seine Bestandteile; Beobachtung der embryologischen Differenzierung aus einfachen Gebilden zu komplizierten Strukturen; Experimentelle Analyse von Arzneimitteln; Korrelierung von morphologischen Läsionen mit funktionellen Störungen ${ }^{33}$. Medizinische Forschung ist besonders dann erfolgreich, so meint Stensen, wenn sie sich auf Mathematik stützt. Eine schlagende Bestätigung dieses Satzes bilden die Erfolge der physiologischen Optik, wo der Strahlengang und das Abbild auf der Retina more geometrico beschrieben werden kann ${ }^{34}$.

Die medizinische Forschung steht in ihren Anfängen, das wissenschaftliche Gebäude der Heilkunde muß erst noch errichtet werden. Dies braucht Zeit. Eine mühevolle nach langen Jahren erst geglückte Entdeckung kann in einem Augenblick demonstriert werden.

Ähnliche Positionen wie Stensen vertrat Malpighi ${ }^{35}$. Er führte aus: Die Naturphänomene - und damit auch biologische Prozesse und therapeutische Mechanismen - folgen notwendigen Naturgesetzen. Dasselbe kann man von den Maschinen unseres Körpers sagen, deren Kenntnis die Basis der 
Heilkunde bildet (lo stesso potiamo dire delle macchine del nostro corpo, che sono la base della medicina) ${ }^{36}$. Die funktionellen Elemente der organischen Maschine, wie die Fäserchen, die Drüschen, die Blutkörperchen usw. sind nur mit dem Mikroskop erforschbar. Die Strömung des Blutes, die Kontraktion der Muskeln gehorchen physikalischen Gesetzen, die Verdauung der Speisen vollzieht sich als chemischer Prozeß. Der Arzt muß daher auch naturwissenschaftliche Methoden beherrschen und dieselben für die Untersuchung des gesunden und kranken Körpers anwenden. Das 17.Jahrhundert, so führt Malpighi aus, hat z. B. in der Forschung der Nierenfunktion bedeutende neue Erkenntnisse gewonnen. Die Alten betrachteten die Nieren als fleischige Eingeweide, die vermöge einer spezifischen Sympathie die serösen Bestandteile aus dem Blut der Hohlvene über die Nierenvenen an sich ziehen. Die modernen Ärzte des 17. Jahrhunderts hingegen betrachteten die Nieren als ein Aggregat von miliaren Drüschen (un aggregato di ghiandole miliari), deren ausführende Gänge das Nierenparenchym zusammensetzen. Das Blut wird durch die Arterien zu den Drüsen getrieben. Dort wird das Blutwasser, mit Salzen und flüchtigen Stoffen gesättigt, aus den Drüsen in die exkretorischen Gänge abgesondert, während das Blut über die Nierenvenen zum IIerzen zurückströmt ${ }^{37}$. Die Kenntnis des exkretorischen Apparates klärt die Pathophysiologie der Nieren und bestimmt prinzipiell auch den therapeutischen Ansatz. Die induktive Methode des großen Bacon wurde von den naturwissenschaftlichen Akademien auf englischem, französischem und deutschem Boden erfolgreich gepflegt ${ }^{38}$. Die wissenschaftich begründete und orientierte Heilkunde übertrifft die rein empirische Medizin durch Einsicht in das innere Getriebe der physiologischen und pathologischen Prozesse, deren Kenntnis allein eine rationelle Pharmakotherapie erlaubt.

Indessen gab es auch skeptische Stimmen. Der große klinische und medizinische Denker Thomas Sydenham bezweifelte, daß die mikroskopische Analyse je die verborgenen Kräfte und Vorgänge im Inneren des Organismus enthüllen könne. Aufgabe des Arztes sei vielmehr die exakte Identifikation von Krankheitsentitäten und die Erprobung spezifischer Pharmaka auf empirischem Wege ${ }^{39}$.

Im Laufe des 17. Jahrhunderts wurde das geschlossene System der klassischen humoralen Pathologie in weiten Bereichen durch die anatomischen und physiologischen Entdeckungen und durch die mechanistische Interpretation des Organismus gesprengt und durch neue pathologische Konzepte ersetzt. Der methodische Dreifuss dieser neuen Pathologie bes- 
tand aus der klinischen Beobachtung, der pathologisch-anatomischen Untersuchung und der experimentellen Physiologie und Pathologie.

Die Apoplexielehre wandelte sich vom humoralen Konzept einer viskösen Verstopfung der Hirnventrikel zur Erklärung des Schlagflusses als einer cerebralen Zirkulationsstörung. Das Pankreas, früher als fleischiges Polster zum Schutze der Bauchgefäße aufgefaßt, wurde durch die anatomische Untersuchung und die physiologische Experimentation als exokrine Drüse beschrieben, deren Pathologie sich auf drei Ebenen abspielte: Erkrankungen der Drüsensubstanz, der exkretorischen Kanäle und des Drüsensekretes. Die Varizenkrankheit erschien als pathologischer Rückfluß venösen Blutes in die durch Wandschwäche erweiterten Varizen. Ein Aszites konnte nicht nur durch Leberverhärtung, sondern auch als Folge von Herzerkrankungen entstehen. Verwachsungen des Herzens mit dem Herzbeutel schwächte die Herzkontraktion, staute das Blut in den großen Gefäßen und erzeugte Aszites.

Die Pathologie entwarf neue Konzepte, die sich in raschem Wandel dem jeweiligen Forschungsstand anpaßten. Aus einer abgeschlossenen Krankheitslehre wurde eine für zukünftige Forschungsergebnisse offene Pathologie.

\section{Anmerkungen}

${ }^{1}$ Mani, Nikolaus: Naturwissenschaftlich-biologische Grundlagenforschung in der Medizin des 17. Jahrhunderts, in: Medizinhistor. Journ. 11 (1976) S. 181-205.

${ }^{2}$ Harvey, William: Exercitatio anatomica de motu cordis et sanguinis in animalibus, Frankfurt 1628, Kp. 16, S. 60-64, S.61.

${ }^{3}$ ibid.: «... et fit paroxysmus, dum calor, scilicet praeternaturalis accensus in corde, inde in totum corpus per arterias diffunditur, una cum materia morbifica, quae eo modo a natura exuperatur, et dissolvitur.»

4 ibid.

5 ibid.: «Cur etiam exterius applicata medicamenta vires intro exercent suas, ac si intro sumpta essent, hinc constet (Colocynthis et Aloe ventrem solvunt, Cantharides urinas movent, Allium plantis pedum alligatum expectorat ...).

${ }^{6}$ De Wale, Jan: Epistolae duae de motu chyli et sanguinis ad Thomam Bartholinum, ed.5., in: Bartholinus, Thomas: Anatomia Reformata, Den Haag 1663, S. 542.

7 De Wale, op. cit. S. 542-543; Walaeus, Johannes: Epistolae duae de motu chyli et sanguinis, Leiden 1640, aus dem Lateinischen ins Deutsche übersetzt von Joseph Gottlieb, Darmstadt 1971, S. 22-23.

${ }^{8}$ Eberhardt-Müller, Ruth: Pankreasforschung im XVII.Jahrhundert, Diss. Bonn 1982.

${ }^{9}$ De Graaf, Regnerus: Tractatus anatomico-medicus de succi pancreatici natura et usu, 
Leiden 1671, in: Opuscula selecta Neerlandicorum de arte medica, Amsterdam 1927, cp.VII: Quibus morbis pancreatis substantia atque illius succus infestari potest (p. 276-293); Eberhardt-Müller, op. cit. S. 120-144.

${ }^{10}$ De Graaf, op. cit., S. 278.

11 De Graaf, op. cit. S.278-282.

12 De Graaf, op. cit. S.287--288.

13 ibid.

${ }^{14}$ De Graaf, op. cit. S. 288: «Obstruuntur unus vel plures pancreatis ductus a materia pituitosa et viscida, una cum succi pancreatici materia, in pancreate secreta et in ductus illos translata atque detenta.

${ }^{15}$ De Graaf, op.cit. S.290: «Peccat idem succus pancreaticus sexto quando sapor eius naturalis mutatur: unde nunc minus acidus, nunc acidior, nune salsus, nunc austerus ... existit.»

16 Wepfer, Johann Jacob: Observationes anatomicae ex cadaveribus eorum, quos sustulit apoplexia, cum exercitatione de eius loco affecto, Schaffhausen 1658 (zit. als Wepfer, Apolexia). Literatur zu Wepfer, s. Mani (Anm.1) S. 196: Mani, N.: Biomedical thought in Glisson's hepatology and in Wepfer's work on apoplexy, in: A celebration of medical history, the fiftieth anniversary of the Johns Hopkins Institute of the History of Medicine, ed. by L.G.Stevenson, Johns Hopkins Un. Pr., Baltimore 1982, S.37-63; Jost, Margret: Die Apoplexielehre Johann Jakob Wepfers (1620-1695), Diss. Bonn 1982.

17 Wepfer, Apoplexia, Vorrede an Leser, dann S.20-21.

18 Wepfer, Apoplexia, S. 82-83.

19 Wepfer, Apoplexia, S. 173.

20 Wepfer, Apoplexia, S. 8-9, 222-241.

21 Wepfer, Apoplexia, S.223.

22 Wepfer, Apoplexia, S. 196-203.

23 Wepfer, Apoplexia, S.205-210.

24 Wepfer, Apoplexia, S. 209.

25 Harvey, William: Exercitationes duae anatomicae de circulatione sanguinis ad Ioannem Riolanum Filium, 1649, in: The circulation of the blood, two anatomical essays by William Harvey, transl. by K.J. Franklin, Oxford 1958, S. 106: «Sicut enim sanorum et boni habitus corporum dissectio plurimum ad philosophiam et rectam physiologiam facit, it a corporum morbosorum et cachecticorum inspectio potissimum ad pathologiam philosophicam.»

${ }^{26}$ Harvey, op.cit. S. 106: «Atque, ausim dicere, plus una dissectio et apertio tabidi, aut morbo aliquo antiquo vel venenato confecti corporis, ad medicinam attulit commodi, quam decem corporum strangulatorum.»

27 Peyer, Johann Conrad: Methodus historiarum anatomico-medicarum exemplo ascitis vitalium organorum vitio ex pericardii coalitu cum corde nati illustrata, ed. sec., in: Peyer, J.C.: Parerga anatomica et medica septem, Amsterdam 1682, parergon 3., S.59-104, insbes. 64-77.

28 Peyer, op.cit. S. 64.

29 Joos-Renfer, Susi, Portmann, Marie-Louise, Buess, Heinrich: Pathologisch-anatomische Beobachtungen bedeutender Schweizer Ärzte 1670-1720, Schwabe, Basel 1961, S. 14-15, 43-46; Sträuli, P.: Johann Conrad Peyer von Schaffhausen und seine «Anatomischmedizinische Methodik», in: Schweiz. Med. Wochschr. 89 (1959) S.825-830, S.828f. 
${ }^{30}$ Stensen, Niels: Discours de Monsieur Stenon sur l'anatomie du cerveau, Paris 1669 (Faks. Ausg. in: Nicolaus Steno's lecture on the anatomy of the brain, introd. by G.Scherz, Copenhagen 1965), S.1-60.

${ }^{31}$ Malpighi, Marcello: Risposta del dottor Marcello Malpighi alla lettera intitolata De recentiorum medicorum studio, in: M. Malpighi, opere scelte a cura di Luigi Belloni, Torino 1967, S. 497-631.

${ }^{32}$ Stensen, op. cit. (Anm. 30) S.1-2, 27, 32-36.

${ }^{33}$ Stensen, op. cit. (Anm. 30) S. 53-57.

${ }^{34}$ Stensen, Niels: Opera philosophica, ed.V.Maar, Vol.2, Copenhagen 1910, S.84 (Widmungsschreiben der Myologie).

${ }^{35}$ Malpighi (Anm. 31).

${ }^{36}$ Malpighi (Anm. 31), S. 512.

${ }^{37}$ Malpighi (Anm. 31), S. 542.

${ }^{38}$ Malpighi (Anm.31), S. 571-572.

${ }^{39}$ Dewhurst, Kenneth: Thomas Sydenham (1624-1689), Berkeley/Los Angeles 1966, S. 85-93.

\section{Summary}

In the course of the seventeenth century classical humoral pathology was partially replaced by new pathological concepts which were based on anatomical dissection, post mortem examinations and experimental physiology and pathology. Some examples illustrate this process. J. J. Wepfer changed radically the pathogenetic concept of apoplexy which was considered to be a circular disease of the brain resulting in ischaemia and not - as previously believed - an obstruction of the ventricles of the brain by viscid humours. R. de Graaf clearly distinguished a threefold pathology of the pancreas based on the new concept of this organ as an excretory gland: Diseases of the glandular substance, lesions of the excretory ducts and chemical alterations of the pancreatic juice. J.C.Peyer explained the genesis of ascites by a concrescence of the heart and pericardium which weakend the contractions of the heart muscle causing stagnation of the blood which resulted in ascites.

Prof.Dr. N. Mani

Medizinhistorisches Institut

Sigmund-Freud-Straße

D-5300 Bonn 1 